\title{
The Effect of Millimeter Wave Source Height on Edge Blocking Diffraction Attenuation
}

\author{
Yongqian $\mathrm{Li}^{1,}$, , Xingrong $\mathrm{Li}^{1, b, ~}{ }^{,}$, Huoer $\mathrm{Zou}^{2, \mathrm{c}}$ and Lei Wang ${ }^{2, \mathrm{~d}}$ \\ ${ }^{1}$ Department of Electronics and Communication Engineering, North China Electric Power University, \\ Baoding 071003, China
${ }^{2}$ The 54th Research Institute of China Electronics Technology Group Corporation, Shijiazhuang 050081, China \\ aliyq@ncepu.edu.cn, bli-xingrong@163.com, c13933121627@139.com, dyifanxiaoli@163.com
}

Keywords: millimeter wave, signal source, edge blockage, diffraction, attenuation.

\begin{abstract}
The geometrical characteristics of radio signals diffracted by edge block and the Vogler multiple edge diffraction function were studied. Three ray method was proposed to model iron board block. In the indoor environment, the diffraction attenuation characteristics of the rectangular iron board blockage were measured by using $45 \mathrm{GHz}$ frequency millimeter wave signal. In the experiments, the height of the transmitting antenna was steered in the range of $0.6-1.7 \mathrm{~m}$ away from the ground, and the diffraction attenuation characteristics of the millimeter wave signal were measured at 8 meters away from the transmitting antenna. The experiment results show that the higher the signal source position is, the smaller the diffraction attenuation is.
\end{abstract}

\section{Introduction}

There are abundant spectrum resources in millimeter wave band, which has attracted wide attention. It will boost the next generation of mobile communication (5G) scale development, to achieve Gigabit wireless data communication possible [1].

The building blocks can cause severe attenuation to millimeter wave signals, and affect the data transmission efficiency. The block properties of buildings in wireless cellular communications were modeled by using stochastic geometry theory, and the relations between coverage probability and building length, building density and base station density were deduced in [2]. Millimeter wave propagation measurements in New York at $28 \mathrm{GHz}$ and $73 \mathrm{GHz}$ were presented by using similar spread spectrum sliding correlator channel sounders, and the signal outage was analyzed $[3,4]$. Some scholars studied the human block effect at $26 \mathrm{GHz}$ and $39 \mathrm{GHz}$ in the laboratory. The experiment results show that the human body block in the indoor environment will cause a very high loss to the transmission link [5,6]. VOGLER and others modeled the edge block of electromagnetic wave, and deduced the theoretical formula of the multiple edge block diffraction [7,8].In the literature [9], a new heuristic approach for multiple edge diffraction modeling based on the uniform theory of diffraction(UTD) was proposed. Several scientists have analyzed the curved edge diffraction and wedge diffraction, and deduced the received electric field in the shadow region [10].

In this paper, the millimeter wave diffraction has been modeled based on theoretical analysis, in which the transmitting antenna was at different heights and blocked by rectangular iron board. A series of channel characteristics measurements were carried out by using $45 \mathrm{GHz}$ millimeter wave under the rectangular iron board block in the laboratory to study the effect of the transmitting antenna height on the diffraction transmission.

\section{Measurement environment and experimental methods}

This experiments were carried out in the laboratory. An arbitrary waveform generator and a spectrum analyzer made by Agilent Co. were utilized in the experiments. The transmitting antenna (TX) and the receiving antenna (RX) are directional horn antennas, and the distance between them was $8 \mathrm{~m}$. The height and width of the iron board block were $1.7 \mathrm{~m}$ and $0.6 \mathrm{~m}$,respectively. During the 
measurements, the iron block was fixed at the position of $1 \mathrm{~m}$ from the TX. The height of the RX was $1.3 \mathrm{~m}$. The TX began to elevate slowly from $0.6 \mathrm{~m}$ away from the ground, and the diffraction loss was measured every 0.1 meters. The diffraction loss were measured 10 times at each position, and the mean value was calculated as the measurement result. The parameters of the measurement system are listed in table 1.

Table 1 The parameters of angle coverage measurement system

\begin{tabular}{|c|c|}
\hline Parameter & Value \\
\hline Carrier & $45 \mathrm{GHz}$ \\
\hline Transmitting power & $0 \mathrm{~dB}$ \\
\hline Antenna gain & $25 \mathrm{~dB}$ \\
\hline Antenna polarization & $10^{\circ}$ \\
\hline Half Power Beam Width of the horn & Vertical polarization \\
\hline
\end{tabular}

\section{VOGLER multiple edge diffraction theory}

The geometry multiple edge diffraction model is shown in figure 1.

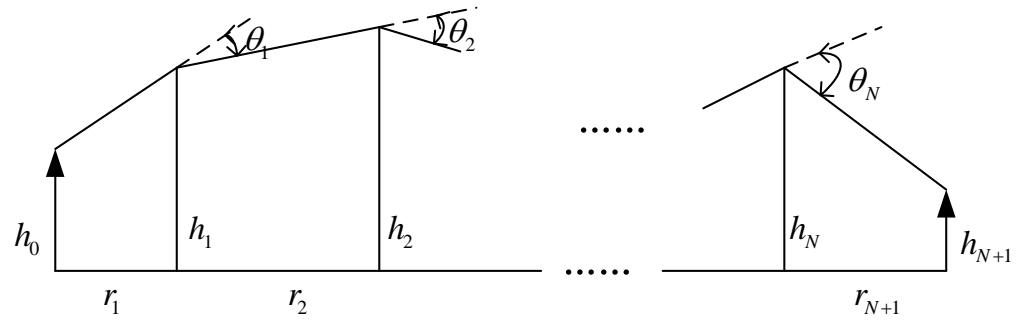

Fig. 1. Geometry for multiple edge diffraction

$\theta_{1}, \theta_{2} \ldots \ldots, \theta_{N}$ are the diffraction angles, $r_{1}, r_{2} \ldots \ldots, r_{N+1}$ are the distance of $N$ knife edges, $h_{1}, h_{2}, \ldots \ldots h_{N}$ are the heights of the knife edges, $h_{0}$ and $h_{N+1}$ are the heights of the TX and the RX. The attenuation of field strength relative to free space, A, over a path of total distance $r_{T}$, and consisting of $N$ knife-edges may be expressed as:

$$
\begin{aligned}
& A=\left(1 / 2^{N}\right) C_{N} e^{\sigma N}(2 / \sqrt{\pi})^{N} \\
& \int_{\beta_{1}}^{\infty} \cdots \int_{\beta_{N}}^{\infty} e^{2 f} \cdot e^{-\left(x_{1}^{2}+x_{2}^{2}+\cdots+x_{N}^{2}\right)} d x_{1} \cdots d x_{N}
\end{aligned}
$$

where

$f=\left\{\begin{array}{l}0, N=1 \\ \sum_{m=1}^{N-1} \alpha_{m}\left(x_{m}-\beta_{m}\right)\left(x_{m+1}-\beta_{m+1}\right) \quad \mathrm{N} \geq 2\end{array}\right.$

$\sigma_{N}=\beta_{1}^{2}+\cdots+\beta_{N}^{2}$

$C_{N}=\left\{\begin{array}{l}1, N=1 \\ {\left[\frac{r_{2} r_{3} \cdots r_{N} r_{T}}{\left(r_{1}+r_{2}\right)\left(r_{2}+r_{3}\right) \cdots\left(r_{N}+r_{N+1}\right)}\right]^{1 / 2} \quad \mathrm{~N} \geq 2}\end{array}\right.$

$r_{T}=r_{1}+r_{2}+\cdots+r_{N+1}$

$\alpha_{m}=\left[\frac{r_{m} r_{m+2}}{\left(r_{m}+r_{m+1}\right)\left(r_{m+1}+r_{m+2}\right)}\right]^{1 / 2}$

$m=1,2, \cdots, N-1$

$\beta_{m}=\theta_{m}\left[\frac{i k r_{m} r_{m+1}}{2\left(r_{m}+r_{m+1}\right)}\right]^{1 / 2}$

$m=1,2, \cdots, N$

When $N=1$, it will be single edge diffraction, (1) and (7) can be simplified as: 


$$
\begin{aligned}
& A=\frac{1}{2} e^{\beta_{1}^{2}} \frac{2}{\sqrt{\pi}} \int_{\beta_{1}}^{\infty} e^{-x^{2}} d x \\
& \beta_{1}=\theta_{1} \sqrt{\frac{i k r_{1} r_{2}}{2\left(r_{1}+r_{2}\right)}}
\end{aligned}
$$

Where, $k=2 \pi / \lambda$ is the wave number, and $i$ is the imaginary number. The detailed process of the formula Is shown in the literature [7].

In actual diffraction problems, it is very useful to subtract a constant from all heights, so that the geometry can be simplified without changing the angle, as shown in figure 2.

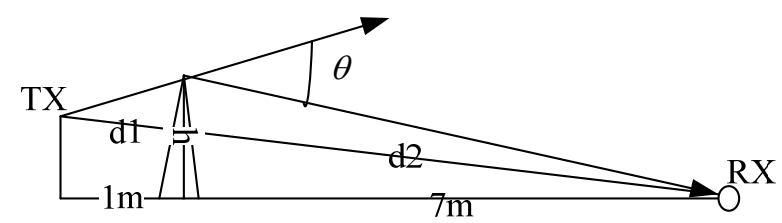

Fig. 2 The equivalent edge diffraction figure

\section{Experiment results and analysis}

The diffraction attenuation of the TX at different heights is shown in figure 3.

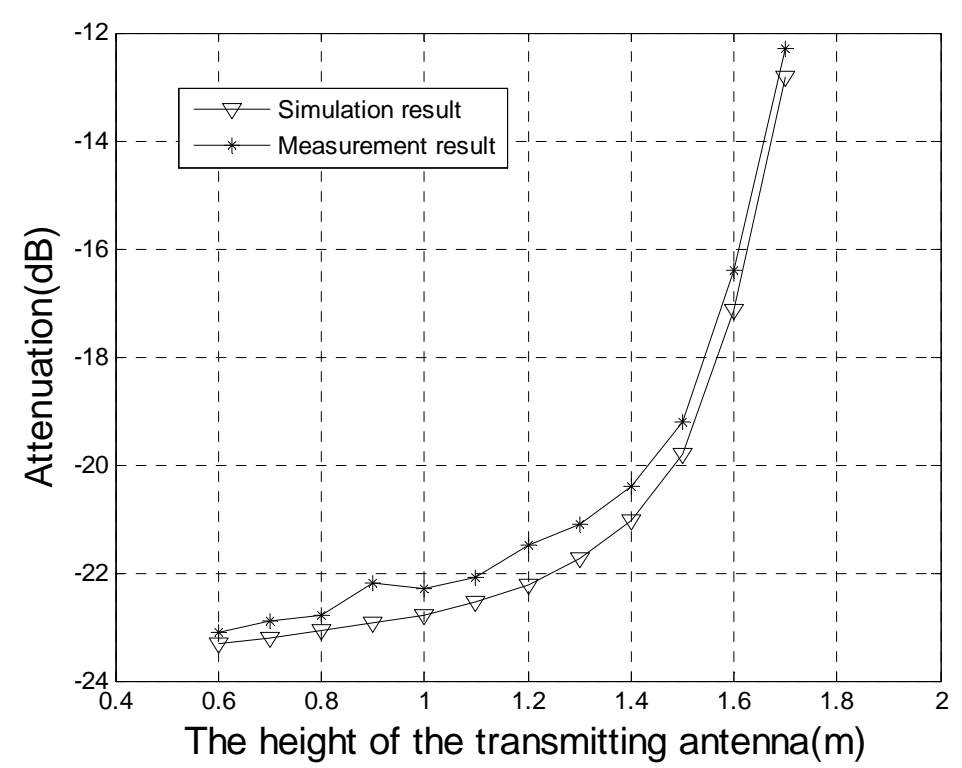

Fig. 3 The diffraction attenuation vs. the height of the TX

From figure 3, the obtained diffraction attenuation agrees well with the simulation results. The received signal increases gradually as the position of the TX elevates, that is, the diffracted attenuation becomes smaller. This is because as the position of the TX elevates, the ray diffraction angle at the top of the iron block becomes smaller, and the signal energy bypassing the iron block is gradually increasing. Due to the influence of the experimental environment and the accuracy of the equipment, there are errors between the measurement results and the simulation results. The error analysis is shown in figure 4 . 


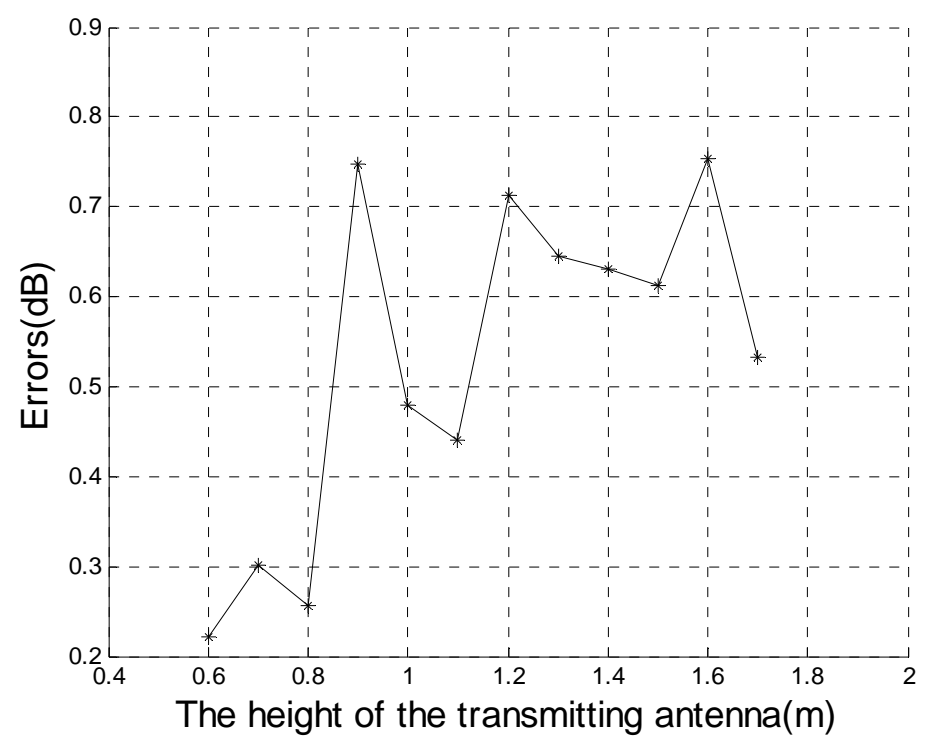

Fig. 4 Errors between the measurement results and the simulation results

The error curve is obtained by subtracting the simulation results from the measurement results. The range of errors are between $0.2-0.75 \mathrm{~dB}$. It can be reduced by improving the system measurement accuracy.

The experiment results show that the height of the TX has a significant influence on the millimeter wave transmission. In the experimental measurement range, the higher the TX is, the better the signal transmission quality is.

\section{Conclusion}

Millimeter wave diffraction loss by the rectangular iron block at $45 \mathrm{GHz}$ was measured. The signal attenuation characteristics were obtained in the case of the height of TX is in $0.6-1.7 \mathrm{~m}$ range and blocked by rectangular iron board. The experiment results show that the higher the TX position is, the smaller the diffraction attenuation is. This conclusion has reference significance for setting up 5G millimeter wave signal source in the environment with edge blocking.

\section{Acknowledgments}

This work is supported by the National Natural Science Foundation of China (Grants No. 61377088) and the Fundamental Research Funds for the Central Universities (Grants No.2015 MS101).

\section{References}

[1] Rappaport T S, Maccartney G, et al, Wideband millimeter-wave propagation measurements and channel models for future wireless communication system design, J. IEEE Trans. on Commun., 63(2015) 3029-3056.

[2] Tianyang Bai, Rahul Vaze, Robert W. Heath, Analysis of Blockage Effects on Urban Cellular Networks, J.IEEE Trans. on Wireless Commun., 13(2014) 5070-5083.

[3] Shuai Nie, George R. MacCartney Jr., Shu Sun, Theodore S. Rappaport,28 GHz and $73 \mathrm{GHz}$ Signal Outage Study for Millimeter Wave Cellular and Backhaul Communications. IEEE ICC 2014, Wireless Communications Symposium, 2014: 4856-4861.

[4] Mustafa Riza Akdeniz, Yuanpeng Liu, Mathew K. Samimi. Millimeter Wave Channel Modeling and Cellular Capacity Evaluation, J.IEEE Journal on Selected Areas in Communications, 32(2014) 1164-1178

[5] Geng S Y, Li X, Wang Q, et al, Research on human blockage effect for indoor $26 \mathrm{GHz}$ mm-wave communications, J. Journal on Communications, 37(2016) 68-73. 
[6] Xiongwen Zhao, Qi Wang, Shu Li, Attenuation by Human Bodies at 26 and 39.5GHz Millimeter Wave Bands, J.IEEE Antennas and Wireless Propagation Letters, 16(2017) 1229-1232.

[7] VOGLER L E, An attenuation function for multiple knife-edge diffraction, J. Radio Science, 17(1982) 1541-1546.

[8] J.Cach Anderson, UTD Multiple-Edge Transition Zone Diffraction, J.IEEE Trans. on Antennas and Propag. ,45(1997) 1093-1097.

[9] C. Tzaras and S. R. Saunders. An improved heuristic UTD solution for multiple-edge transition zone diffraction [J]. IEEE Trans. Antennas and Propag., 49(2001) 1678-1682.

[10] R. G. Kouyoumjian , P. H. Pathak, A Uniform Geometrical Theory of Diffraction for an Edge in a Perfectly Conducting Surface, J. Proceeding of The IEEE, 62(1974) 1448-1461. 